\title{
Emergence of topological and strongly correlated ground states in trapped Rashba spin-orbit-coupled Bose gases
}

\author{
B. Ramachandhran, ${ }^{1} \mathrm{Hui} \mathrm{Hu},{ }^{2}$ and $\mathrm{Han} \mathrm{Pu}^{1}$ \\ ${ }^{1}$ Department of Physics and Astronomy, Rice University, Houston, Texas 77005, USA \\ ${ }^{2}$ ARC Centres of Excellence for Quantum-Atom Optics and Centre for Atom Optics and Ultrafast Spectroscopy, \\ Swinburne University of Technology, Melbourne 3122, Australia
}

(Received 3 January 2013; published 26 March 2013)

\begin{abstract}
We theoretically study an interacting few-body system of Rashba spin-orbit-coupled two-component Bose gases confined in a harmonic trapping potential. We solve the interacting Hamiltonian at large Rashba coupling strengths using an exact-diagonalization scheme, and obtain the ground-state phase diagram for a range of interatomic interactions and particle numbers. At small particle numbers, we observe that the bosons condense to an array of topological states with $n+1 / 2$ quantum angular momentum vortex configurations, where $n=$ $0,1,2,3, \ldots$. At large particle numbers, we observe two distinct regimes: at weaker-interaction strengths, we obtain ground states with topological and symmetry properties that are consistent with mean-field theory computations; at stronger-interaction strengths, we report the emergence of strongly correlated ground states.
\end{abstract}

DOI: 10.1103/PhysRevA.87.033627 PACS number(s): 03.75.Mn, 05.30.Jp, 71.70.Ej, 71.45.Gm

\section{INTRODUCTION}

Ultracold atomic gases offer an exceptional platform to explore many-body quantum phenomena due to outstanding experimental control over interatomic interactions, system geometry, density, and purity [1]. Numerous research groups have, for example, successfully demonstrated the manifestation of few-body bound states and superfluid states in Bose and Fermi gases in trapped atom experiments [2,3]. Furthermore, phenomenal experimental progress has been achieved with atomic gases loaded in optical lattices to emulate traditionally condensed-matter phenomena such as superfluidinsulator transition, antiferromagnetism, and frustrated manybody systems [4-6]. However, due to the neutral nature of atomic gases, most experimental systems have been limited to exploring quantum phenomena that would occur in the absence of electromagnetic fields. Recently, even this limitation was overcome when laser fields were used to successfully generate effective magnetic and electric fields in neutral atoms [7]. The introduction of (synthetic) gauge fields in ultracold neutral atomic systems has thus opened the possibility of exploring a whole new set of phenomena that would manifest in the presence of Abelian and non-Abelian vector potentials [8].

In the presence of synthetic gauge fields in trapped ultracold bosonic systems, experimental evidence for spin-orbit (SO) coupling with equal Rashba and Dresselhaus-type strengths was reported in a seminal paper [9]. Recently, commendable experimental progress has also been achieved towards simulating SO coupling in ultracold fermionic systems [10], a phenomenon critical to the simulation of certain topologically insulating states in condensed-matter systems [11]. In the presence of SO coupling, a generic Hamiltonian may be broadly classified in two classes: (a) one that breaks $\mathcal{T}$ (time-reversal) symmetry, and which can be shown to be gauge equivalent to a Hamiltonian in the combined presence of Abelian and non-Abelian vector potentials (For example, authors in Ref. [12] consider an SO-coupling Hamiltonian in the presence of a real (Abelian) magnetic field and attempt to simulate the physics of traditional quantum Hall systems.); and (b) one that preserves $\mathcal{T}$ symmetry, and which can be shown to be gauge equivalent to a Hamiltonian in a pure non-Abelian vector potential. In this work, we study an SO-coupling Hamiltonian of the latter class, and discuss the emergence of ground states with unique topological and correlation properties.

In this paper, we study an interacting few-body system of two-component Bose gases confined in a two-dimensional (2D) isotropic harmonic trapping potential with Rashba SO coupling. The paper is organized as follows: In Sec. II, we outline the model Rashba SO-coupling Hamiltonian and discuss various symmetries. We show that the Hamiltonian is gauge equivalent to particles subject to a pure non-Abelian vector potential that preserves $\mathcal{T}$ symmetry. Then, we consider the noninteracting limit of this Hamiltonian, and discuss single-particle solutions at small and large SO-coupling strengths. We proceed to discuss the implementation of an exact-diagonalization (ED) scheme to obtain the low-energy eigenstates of the interacting Hamiltonian in the regime of interest to us-at large SO-coupling strengths. Then, we introduce various analysis techniques, namely, energy spectrum, density distribution, single-particle density matrix, paircorrelation function, reduced wave function, entanglement spectrum, and entanglement entropy. Each technique would offer its unique perspective to the overall understanding of the ground-state properties.

In Sec. III, we discuss the phase diagram and analyze the ground-state properties of the interacting Hamiltonian at different particle numbers $N$, and at varied interatomic interaction strengths. At small particle numbers with $N=2$, we illustrate the unique topological and symmetry properties of ground states. In the relatively large particle number scenario with $N=8$, we observe that the ground states fall into two distinct regimes: (a) at weak-interaction strengths (mean-field-like regime), we observe ground states with topological and symmetry properties that are also obtained via mean-field theory computations; and (b) at intermediate- to strong-interaction strengths (strongly correlated regime), we report the emergence of strongly correlated ground states. We proceed to illustrate the topological, symmetry, and 
strong-correlation properties of these ground states. Finally, in Sec. IV, we summarize and present concluding remarks.

\section{THEORETICAL FRAMEWORK}

\section{A. System under study}

We study a two-component Bose gas confined in a $2 \mathrm{D}$ isotropic harmonic trapping potential: $V(\rho)=M \omega_{\perp}^{2}\left(x^{2}+\right.$ $\left.y^{2}\right) / 2=M \omega_{\perp}^{2} \rho^{2} / 2$. We consider the Rashba SO-coupling term, which couples pseudo-spin-1/2 degree of freedom and linear momentum, of the form $\mathcal{V}_{\text {SO }}=-i \lambda_{R}\left(\hat{\sigma}_{x} \partial_{y}-\hat{\sigma}_{y} \partial_{x}\right)$, where $\lambda_{R}$ is the Rashba SO-coupling strength and $\hat{\sigma}_{x, y, z}$ are $2 \times 2$ Pauli matrices. The model Hamiltonian for the interacting system is then given by $\mathcal{H}=\int d \mathbf{r}\left[\mathcal{H}_{0}+\mathcal{H}_{\text {int }}\right]$,

$$
\begin{gathered}
\mathcal{H}_{0}=\Psi^{\dagger}\left[-\frac{\hbar^{2} \nabla^{2}}{2 M}+V(\rho)+\mathcal{V}_{\text {SO }}-\mu\right] \Psi, \\
\mathcal{H}_{\text {int }}=(g / 2) \sum_{\sigma=\uparrow, \downarrow} \Psi_{\sigma}^{\dagger} \Psi_{\sigma}^{\dagger} \Psi_{\sigma} \Psi_{\sigma}+g_{\uparrow \downarrow} \Psi_{\uparrow}^{\dagger} \Psi_{\uparrow} \Psi_{\downarrow}^{\dagger} \Psi_{\downarrow},
\end{gathered}
$$

where $\mathbf{r}=(x, y)$ and $\Psi=\left[\Psi_{\uparrow}(\mathbf{r}), \Psi_{\downarrow}(\mathbf{r})\right]^{T}$ denotes the spinor Bose field operators. The chemical potential $\mu$ is to be determined by the total number of bosons $N$ (i.e., $\int d \mathbf{r} \Psi^{\dagger} \Psi=$ $N)$. For simplicity, we have assumed that the intracomponent interaction strengths are equal, so that $g_{\uparrow \uparrow}=g_{\downarrow \downarrow}=g$. The Hamiltonian is invariant under symmetry operations associated with the antiunitary time-reversal operator $\mathcal{T}=i \hat{\sigma}_{y} \mathcal{C}$ and the unitary parity operator $\mathcal{P}=\hat{\sigma}_{z} \mathcal{I}$, where $\mathcal{C}$ and $\mathcal{I}$ perform complex-conjugation and spatial-inversion operations, respectively. The Hamiltonian is also invariant under the combined $\mathcal{P} \mathcal{T}$ operator, which is unitary since operators $\mathcal{P}$ and $\mathcal{T}$ anticommute, i.e., since $[\mathcal{P}, \mathcal{T}]_{+}=0$. We further note that the Rashba SO-coupling term breaks inversion symmetry.

In experiments, the two dimensionality can be realized by imposing a strong harmonic potential $V(z)=M \omega_{z}^{2} z^{2} / 2$ along the axial direction in such a way that $\mu, k_{B} T \ll \hbar \omega_{z}$. For the realistic case of ${ }^{87} \mathrm{Rb}$ atoms, the interaction strengths can be calculated from the two $s$-wave scattering lengths $a \simeq 100 a_{B}$ and $a_{\uparrow \downarrow}$, using $g=\sqrt{8 \pi}\left(\hbar^{2} / M\right)\left(a / a_{z}\right)$ and $g_{\uparrow \downarrow}=$ $\sqrt{8 \pi}\left(\hbar^{2} / M\right)\left(a_{\uparrow \downarrow} / a_{z}\right)$, respectively. Here, $a_{z}=\sqrt{\hbar /\left(M \omega_{z}\right)}$ is the characteristic oscillator length in the $z$ direction, and $a_{B}$ is the atomic Bohr radius. Note that throughout this work, we consider interaction strengths such that $a_{z} \gg a, a_{\uparrow \downarrow}$. In another possible regime of strong interactions where $a_{z} \simeq a, a_{\uparrow \downarrow}$, one needs to include confinement-induced resonance in the calculation of 2D interaction strengths $g$ and $g_{\uparrow \downarrow}$ [13].

In harmonic traps, it is natural to use the trap units; that is, to take $\hbar \omega_{\perp}$ as the unit for energy, and the harmonic oscillator length $a_{\perp}=\sqrt{\hbar /\left(M \omega_{\perp}\right)}$ as the unit for length. This is equivalent to setting $\hbar=k_{B}=M=\omega_{\perp}=1$. For the SO coupling, we introduce an SO-coupling length $a_{\lambda}=\hbar^{2} /\left(M \lambda_{R}\right)$ and, consequently, define a dimensionless SO-coupling strength $\lambda_{\text {SO }}=a_{\perp} / a_{\lambda}=\sqrt{\left(M / \hbar^{3}\right)} \lambda_{R} / \sqrt{\omega_{\perp}}$. In a recent experiment [9], a spinor (spin-1) Bose gas of ${ }^{87} \mathrm{Rb}$ atoms with $F=1$ ground-state electronic manifold is used to create SO coupling, where two internal "spin" states are selected from this manifold and labeled as pseudo-spin-up and pseudo-spin-down. This gives an effective spin-1/2 Bose gas. In this SO-coupled spin-1/2 BEC, $\lambda_{\text {SO }}$ is about 10 . In a typical experiment for 2D spin-1/2 ${ }^{87} \mathrm{Rb}$ Bose-Einstein condensates (BECs) [14], the interatomic interaction strengths are about $g(N-1) \approx$ $g_{\uparrow \downarrow}(N-1)=10^{2} \sim 10^{3}\left(\hbar \omega_{\perp} a_{\perp}^{2}\right)$. These coupling strengths, however, can be precisely tuned by properly choosing the parameters of the laser fields that lead to the harmonic confinement and the SO coupling.

\section{B. Gauge-equivalent form of $\mathcal{H}_{\mathbf{0}}$}

A generic single-particle Hamiltonian may be written in the form $\mathcal{H}_{g}=(\mathbf{p}-\mathbf{A})^{2} / 2 M$, where $\mathbf{p}=\hbar \mathbf{k}$ is the particle momentum and $\mathbf{k}$ is the wave vector. The vector potential $\mathbf{A}$ may possibly have components in both physical space and spin space. Depending upon the commutation properties of the components of $\mathbf{A}$, we may hence have an Abelian-type or non-Abelian-type vector potential. The primary motivation behind deriving a gauge-equivalent form is to map our model Hamiltonian $\mathcal{H}_{0}$ onto $\mathcal{H}_{g}$, and hence derive the nature of $\mathbf{A}$. It is conceivable that depending upon the nature of $\mathcal{H}_{0}, \mathbf{A}$ could be comprised of purely Abelian components or purely non-Abelian components, or a combination of both.

In order to map $\mathcal{H}_{0}$ onto $\mathcal{H}_{g}$, it suffices to compare $\mathcal{H}_{g}$ with the terms $-\hbar^{2} \nabla^{2} / 2 M-i \lambda_{R}\left(\hat{\sigma}_{x} \partial_{y}-\hat{\sigma}_{y} \partial_{x}\right)$ in $\mathcal{H}_{0}$. The latter terms may actually be rewritten as $|\mathbf{p}|^{2} / 2 M+\lambda_{R}\left(\hat{k}_{y} \hat{\sigma}_{x}-\right.$ $\hat{k}_{x} \hat{\sigma}_{y}$ ). For a two-component Bose gas confined in a $2 \mathrm{D}$ isotropic harmonic trap, we have a two-component vector potential A, with $A_{x}, A_{y}$ being $2 \times 2$ matrices. Comparing $\mathcal{H}_{0}$ with $\mathcal{H}_{g}$, we expect $A_{x} \propto \hat{\sigma}_{y}$ and $A_{y} \propto-\hat{\sigma}_{x}$. Specifically, it can be shown that the vector potential is $\mathbf{A}=\left(A_{x}, A_{y}, 0\right)=$ $\left(\hbar M \omega_{\perp}\right)^{1 / 2} \lambda_{\mathrm{SO}}\left(\hat{\sigma}_{y},-\hat{\sigma}_{x}, 0\right)$. In trap units, we then simply have $\mathbf{A}=\lambda_{\mathrm{SO}}\left(\hat{\sigma}_{y},-\hat{\sigma}_{x}, 0\right)$. The term involving $|\mathbf{A}|^{2}$ is a constant, and can be gauged out without loss of generality. Therefore, the strength of the non-Abelian vector potential proportionally determines the strength of SO coupling. It is further evident that $\left[A_{x}, A_{y}\right] \neq 0$, and that $\mathbf{A}$ is a pure non-Abelian vector potential. Furthermore, the $\mathcal{T}$ operator commutes with the SOcoupling term $\lambda_{R}\left(\hat{k}_{y} \hat{\sigma}_{x}-\hat{k}_{x} \hat{\sigma}_{y}\right)$. In essence, the model Rashba SO-coupling Hamiltonian in Eq. (1) is gauge equivalent to particles subject to a pure non-Abelian vector potential that preserves $\mathcal{T}$ symmetry. Proposals to realize vector potentials of similar forms have been addressed by multiple groups [8,15-17].

\section{Single-particle solutions}

We solve the model Hamiltonian $\mathcal{H}$ in the absence of interatomic interactions and obtain the single-particle solutions. Rewriting the $\mathcal{H}_{0}$ component in Eq. (1), the single-particle wave function $\phi(\mathbf{r})=\left[\phi_{\uparrow}(\mathbf{r}), \phi_{\downarrow}(\mathbf{r})\right]^{T}$ with energy $\epsilon$ is given by

$$
\left[\begin{array}{cc}
\mathcal{H}_{\mathrm{osc}} & -i \lambda_{R}\left(\partial_{y}+i \partial_{x}\right) \\
-i \lambda_{R}\left(\partial_{y}-i \partial_{x}\right) & \mathcal{H}_{\mathrm{osc}}
\end{array}\right]\left[\begin{array}{l}
\phi_{\uparrow} \\
\phi_{\downarrow}
\end{array}\right]=\epsilon\left[\begin{array}{l}
\phi_{\uparrow} \\
\phi_{\downarrow}
\end{array}\right],
$$

where $\mathcal{H}_{\mathrm{osc}} \equiv-\hbar^{2} \nabla^{2} /(2 M)+V(\rho)$. In polar coordinates $(\rho, \varphi)$, we have $-i\left(\partial_{y} \pm i \partial_{x}\right)=e^{\mp i \varphi}[ \pm \partial / \partial \rho-(i / \rho) \partial / \partial \varphi]$. The single-particle wave function takes the form

$$
\phi_{m}(\mathbf{r})=\left[\begin{array}{c}
\phi_{\uparrow}(\rho) \\
\phi_{\downarrow}(\rho) e^{i \varphi}
\end{array}\right] \frac{e^{i m \varphi}}{\sqrt{2 \pi}},
$$


with well-defined total angular momentum $j_{z}$, which is a sum of orbital and spin angular momenta. In general, we may denote the energy spectrum as $\epsilon_{n m}$, where $n=(0,1,2, \ldots)$ is the quantum number for the transverse (radial) direction.

The single-particle wave function $\phi_{m}(\mathbf{r})$ is an eigenstate of the unitary $\mathcal{P}$ operator,

$$
\mathcal{P} \phi_{m}(\mathbf{r})=\sigma_{z}(-1)^{m}\left[\begin{array}{c}
\phi_{\uparrow}(\rho) \\
-\phi_{\downarrow}(\rho) e^{i \varphi}
\end{array}\right] \frac{e^{i m \varphi}}{\sqrt{2 \pi}}=(-1)^{m} \phi_{m}(\mathbf{r}) .
$$

The $\mathcal{T}$ symmetry preserved by the Hamiltonian results in a twofold degeneracy (Kramer doublet) of the energy spectrum: any eigenstate $\phi(\mathbf{r})=\left[\phi_{\uparrow}(\mathbf{r}), \phi_{\downarrow}(\mathbf{r})\right]^{T}$ is degenerate with its time-reversal partner $\mathcal{T} \phi(\mathbf{r})=\left[\phi_{\downarrow}^{*}(\mathbf{r}),-\phi_{\uparrow}^{*}(\mathbf{r})\right]^{T}$. This symmetry is preserved even in the presence of interatomic interactions, as the terms in interacting Hamiltonian $\mathcal{H}_{\text {int }}$ are $\mathcal{T}$ invariant. The superposition state of $\phi_{m}(\mathbf{r})$ and its time-reversal partner state is an eigenstate of the unitary $\mathcal{P} \mathcal{T}$ operator,

$$
\mathcal{P} \mathcal{T}\left[\phi_{m}(\mathbf{r})+\mathcal{T} \phi_{m}(\mathbf{r})\right]=(-1)^{m+1}\left[\phi_{m}(\mathbf{r})+\mathcal{T} \phi_{m}(\mathbf{r})\right] .
$$

We solve the single-particle spectrum by adopting a numerical basis-expansion method, details of which are outlined in our earlier work [18]. In Fig. 1, we show wave functions of single-particle eigenstates at representative values of small and large SO-coupling strengths. It is evident that a larger SOcoupling strength leads to increased oscillations and increased localization at radii determined by $|m|$ in the radial direction. Corresponding wave functions $\phi_{\downarrow}(\rho)$ also have similar characteristics. In Fig. 2, we show the energy spectrum for single-particle states at small and large SO-coupling strengths. From Fig. 2(a), it is evident that the energy spectrum is strongly dispersive in $m$ at small SO-coupling strengths, with a large overlap between the energies of single-particle states with different radial quantum number $n$. Qualitatively, the energy spectrum at small SO-coupling strengths may be understood as a weak perturbation of the harmonic-oscillator energy levels of the two pseudospin components. On the other hand, we observe from Fig. 2(b) that the energy spectrum is weakly dispersive or nearly flat in $m$ at large SO-coupling strengths. For the range of $m$ shown here, there is no overlap between the energies

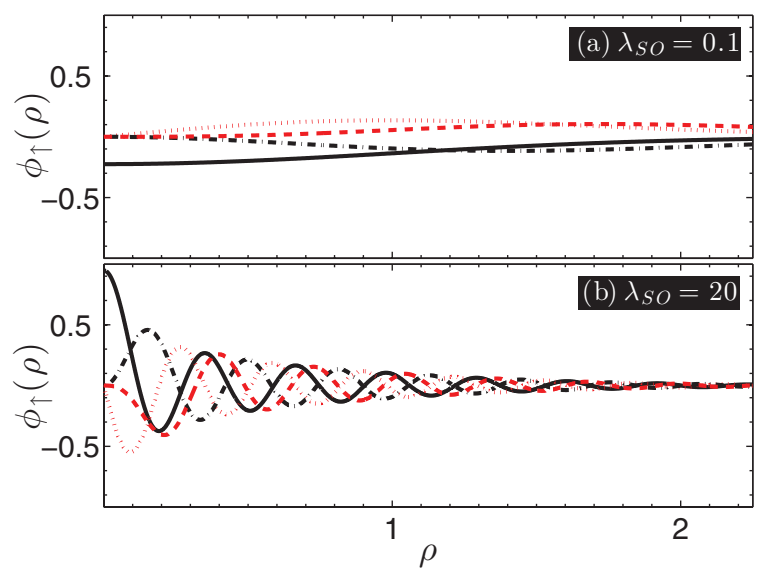

FIG. 1. (Color online) Plots (a) and (b) show wave functions $\phi_{\uparrow}(\rho)$ of single-particle states in the $n=0$ manifold at small and large SO-coupling strengths, respectively. $m=0$ (solid black line), $m=1$ (dotted red line), $m=2$ (dash-dotted black line), and $m=3$ (dashed red line).

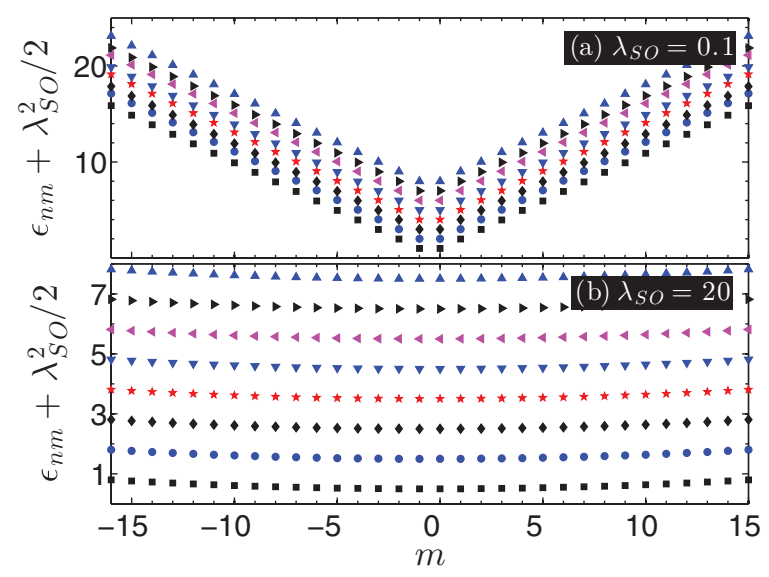

FIG. 2. (Color online) Plots (a) and (b) show the energy spectrum of single-particle states at small and large SO-coupling strengths, respectively: $n=0 \rightarrow 7$ (bottom $\rightarrow$ top) and $m=-16 \rightarrow+15$. While energies of states within each $n$ are represented by a specific symbol, it is evident that states with higher $n$ have progressively higher energies.

of single-particle states belonging to different radial quantum numbers $n$, i.e., each $n$ manifold represents single-particle states labeled by their azimuthal angular momenta $m$ with no overlap with adjacent $n$ manifolds. Furthermore, the harmonic trapping potential may be qualitatively understood as a weak perturbation to the energy spectrum at large SO-coupling strengths of the corresponding translationally invariant system.

The localized nature of the wave functions in Fig. 1(b) and the weakly dispersive nature of the single-particle energy spectrum in Fig. 2(b) are characteristics that justify a comparison of the single-particle basis states at large SO-coupling strengths with 2D Landau level (LL) structures in magnetic fields. In Ref. [16], the authors discuss the mapping between a $\mathcal{H}_{0}$ and 2D LL Hamiltonian in a rigorous fashion and generalize the terminology of LLs as "topological single-particle level structures labeled by angular momentum quantum numbers with flat or nearly flat spectra." Making use of this generalization, we term the $n=0$ manifold as the lowest LL structure (LLL), the $n=1$ manifold as the next highest LL, and so on. As seen in Fig. 2(b), the radial quantization generates energy gaps between adjacent LLs of the order of trap energy $\hbar \omega_{\perp}$, i.e., of the order of unity in trap units.

To summarize, we emphasize that the generalized LLs discussed here are created by a truly non-Abelian vector potential, i.e., in the absence of any real (Abelian) magnetic fields. The strength of Rashba SO-coupling strength and, in turn, the flatness of the single-particle energy spectra can be experimentally controlled by using laser fields. At large SO-coupling strengths, as shown for $\lambda_{\mathrm{SO}}=20$, we obtain a nearly flat single-particle energy spectra. In a noninteracting two-component Bose gas, quantum statistics obviates the occurrence of correlated states in a spectra that is not perfectly flat, due to potential condensation of all the particles in the lowest-energy single-particle states, identified by $j_{z}= \pm 0.5$, of the LLL ( $n=0$ manifold). However, in the presence 
of interparticle interactions, nearly flat energy spectra is sufficiently enabled to act as an interesting playground to allow for the emergence of strongly correlated ground states. We now proceed to introduce the ED scheme to solve the interacting Rashba SO-coupled Hamiltonian at large SOcoupling strengths.

\section{Interacting few-body problem: Exact-diagonalization scheme}

We solve the interacting Rashba SO-coupled Hamiltonian $\mathcal{H}$ in Eqs. (1) and (2) within the configuration-interaction, alias exact-diagonalization, scheme. In this scheme, we expand the interacting many-body Hamiltonian in an appropriate single-particle basis (configuration) to obtain the solution. The solution becomes exact when we consider an infinite number of single-particle states. With $N$ bosons and $M$ single-particle states in the basis, the dimension of Hilbert space is $D=(N+$ $M-1) ! / N !(M-1)$ !. With $M=24$, for example, $D=300$ for $N=2$, and $D=7888725$ for $N=8$. The dimension of Hilbert space grows dramatically with system size and hence, for practical purposes, we limit our configuration to a finite size. We observe that the solution becomes essentially exact when we consider a sufficient number of single-particle states. To solve the problem at hand, it is convenient to work with the SO single-particle basis,

$$
\Phi(\mathbf{r})=\sum_{n m}\left[\begin{array}{l}
\phi_{\uparrow n m}(\mathbf{r}) \\
\phi_{\downarrow n m}(\mathbf{r})
\end{array}\right] a_{n m} \equiv \sum_{i \equiv n m}\left[\begin{array}{l}
\phi_{\uparrow i}(\mathbf{r}) \\
\phi_{\downarrow i}(\mathbf{r})
\end{array}\right] a_{i},
$$

where the field operator $a_{i}$ is related to the single-particle state $\left[\phi_{\uparrow n m}(\mathbf{r}), \phi_{\downarrow n m}(\mathbf{r})\right]^{T}$. Then, Eqs. (1) and (2) simply become

$$
\mathcal{H}=\sum_{i} \epsilon_{i} a_{i}^{\dagger} a_{i}+\sum_{i j k l} V_{i j k l} a_{i}^{\dagger} a_{j}^{\dagger} a_{k} a_{l},
$$

where $(i, j, k, l)$ collectively denotes $(n, m)$, and $V_{i j k l}=$ $(g / 2)\left[V_{i j k l}^{\uparrow \uparrow}+V_{i j k l}^{\downarrow \downarrow}\right]+g_{\uparrow \downarrow} V_{i j k l}^{\uparrow \downarrow}$ with

$$
\begin{aligned}
V_{i j k l}^{\uparrow \uparrow} & =\int d \mathbf{r} \phi_{\uparrow i}^{*}(\mathbf{r}) \phi_{\uparrow j}^{*}(\mathbf{r}) \phi_{\uparrow k}(\mathbf{r}) \phi_{\uparrow l}(\mathbf{r}), \\
V_{i j k l}^{\downarrow \downarrow} & =\int d \mathbf{r} \phi_{\downarrow i}^{*}(\mathbf{r}) \phi_{\downarrow j}^{*}(\mathbf{r}) \phi_{\downarrow k}(\mathbf{r}) \phi_{\downarrow l}(\mathbf{r}), \\
V_{i j k l}^{\uparrow \downarrow} & =\int d \mathbf{r} \phi_{\uparrow i}^{*}(\mathbf{r}) \phi_{\downarrow j}^{*}(\mathbf{r}) \phi_{\uparrow k}(\mathbf{r}) \phi_{\downarrow l}(\mathbf{r}) .
\end{aligned}
$$

We perform the ED calculation in Fock space and the Hamiltonian $\mathcal{H}$ can be written as a matrix of dimension $D^{2}$, naively accounting for the possibility of intercoupling every Fock state [19]. It is clear from the single-particle solutions discussed in Eq. (3) that the single-particle term $\epsilon_{i} a_{i}^{\dagger} a_{i}$ contributes only to diagonal entries of the Hamiltonian matrix, while the interaction term $V_{i j k l} a_{i}^{\dagger} a_{j}^{\dagger} a_{k} a_{l}$ contributes to off-diagonal entries as well. The enumeration of off-diagonal entries can be enormously simplified by accounting for a symmetry preserved by $\mathcal{H}$ : conservation of total angular momentum $J_{z}=\sum_{N} j_{z}$, as readily seen from Eq. (6). If an entry $V_{i j k l}$ is to be nonzero, we must have $m_{i}+m_{j}=m_{k}+m_{l}$ in Eq. (7). Using only the radial wave function, we have $\left(\right.$ provided $\left.m_{i}+m_{j}=m_{k}+m_{l}\right)$

$$
\begin{aligned}
V_{i j k l}^{\uparrow \uparrow} & =\frac{1}{2 \pi} \int_{0}^{\infty} \rho d \rho \phi_{\uparrow i}(\rho) \phi_{\uparrow j}(\rho) \phi_{\uparrow k}(\rho) \phi_{\uparrow l}(\rho), \\
V_{i j k l}^{\downarrow \downarrow} & =\frac{1}{2 \pi} \int_{0}^{\infty} \rho d \rho \phi_{\downarrow i}(\rho) \phi_{\downarrow j}(\rho) \phi_{\downarrow k}(\rho) \phi_{\downarrow l}(\rho), \\
V_{i j k l}^{\uparrow \downarrow} & =\frac{1}{2 \pi} \int_{0}^{\infty} \rho d \rho \phi_{\uparrow i}(\rho) \phi_{\downarrow j}(\rho) \phi_{\uparrow k}(\rho) \phi_{\downarrow l}(\rho) .
\end{aligned}
$$

This enables one to visualize the Hamiltonian in blockdiagonal form, i.e., each block is a manifold comprised of Fock states with a fixed $J_{z}$. Hence, the term $V_{i j k l} a_{i}^{\dagger} a_{j}^{\dagger} a_{k} a_{l}$ can only couple states within the same manifold, therefore resulting in a sparse Hamiltonian matrix. We solve this sparse matrix to identify the low-energy states of the system.

As discussed in Sec. II A, the Hamiltonian $\mathcal{H}$ preserves $\mathcal{T}$ symmetry. In a certain LL, the energies of states labeled $j_{z}$ and $-j_{z}$ are equal and, hence, we need to consider both positive and negative angular momentum states in the single-particle configuration. This has two major implications: (a) computational intensity increases tremendously, and (b) a given configuration would never be sufficient to obtain a complete $J_{z}$ manifold, where all contributing single-particle states are included. We note here that the latter issue does not arise when the Hamiltonian breaks $\mathcal{T}$ symmetry, as in studies of rotating trapped gases or gases subject to real magnetic fields [20,21]. In these studies, it was sufficient to consider only positive $j_{z}$ states and, hence, obtain complete $J_{z}$ manifolds. In the limit of large SO-coupling strengths, if the interaction strengths are such that the energy contribution from $H_{\text {int }}$ is less than unity (in trap units), we may restrict ourselves to the lowest $n=0$ manifold. Within this LLL approximation, we may consider a sufficient number of single-particle eigenstates to obtain essentially exact low-energy eigenstates.

\section{E. Analysis techniques}

The ED scheme enables us to solve the Rashba SO-coupled Hamiltonian $\mathcal{H}$ and obtain the ground-state phase diagram at various interaction strengths and particle numbers. The ground states have interesting topological, symmetry, and strong-correlation properties. Here, we outline the details of various techniques that we use to analyze these properties.

\section{Energy spectrum}

The first step in our analysis is to identify the total angular momentum manifold $J_{z}$ to which the ground state belongs. As discussed earlier, the Hamiltonian matrix has a block-diagonal form, with each block identified by its unique $J_{z}$ value. It is evident that each of these blocks can essentially be diagonalized independently. The energy spectrum is comprised of energy eigenvalues from each block, and the lowest eigenvalue and its corresponding $J_{z}$ may be readily associated with the ground state. Degeneracies in the energy spectrum naturally reflect the degeneracies in the ground state. For example, a typical energy spectrum plot is shown in Fig. 3.

The dimension of Fock space in the ground state $J_{z}$ manifold will be much smaller when compared to the Hilbertspace dimension $D$. For a given parameter set, once we identify 

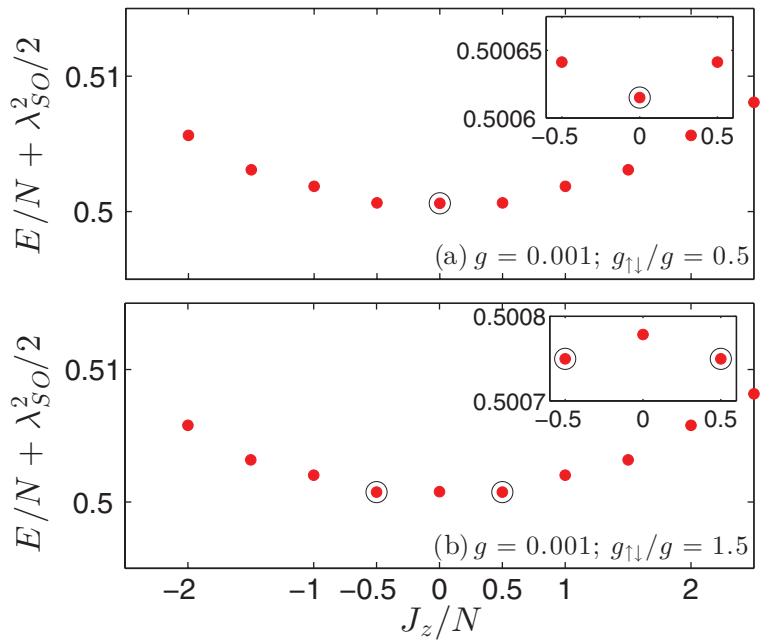

FIG. 3. (Color online) Energy spectrum for extremely weakinteraction strengths with $\lambda_{\text {SO }}=20$ and $N=2$. Here, each marker (red) represents the lowest-energy eigenvalue of a specific block diagonal with a fixed value of $J_{z}$. Since energy eigenvalues are very close, we identify the ground-state energies by circled (black) markers and, further, show the zoomed-in plots in the inset.

the ground-state $J_{z}$ manifold, we can extract the coefficients of all Fock states from the corresponding eigenvector. In essence, we may then represent the ground-state wave function as a sum of all contributing Fock states: $\Psi_{G}=\sum_{p=1}^{n_{d}} \alpha_{p} \Phi_{p}$, where $n_{d}$ is the dimension of the ground-state $J_{z}$ manifold and $\alpha_{p}$ is the coefficient of the Fock state $\Phi_{p}$. As discussed in Sec. II A, the interacting Hamiltonian $\mathcal{H}$ is invariant under two unitary symmetry operations, $\mathcal{P}$ and $\mathcal{P} \mathcal{T}$. With the knowledge of ground-state wave function $\Psi_{G}$, we are now equipped to determine if the ground state is an eigenstate of the $\mathcal{P}$ or $\mathcal{P} \mathcal{T}$ operator.

\section{Density distribution and single-particle density matrix}

With the knowledge of $\Psi_{G}$, we are equipped to extract various properties of the ground state. We derive the density distribution from the expectation value of the single-particle density operator, written in second-quantized form as

$$
\hat{\rho}(\mathbf{r})=\sum_{i j}\left\langle\phi_{i}\left(\mathbf{r}^{\prime}\right)\left|\delta\left(\mathbf{r}-\mathbf{r}^{\prime}\right)\right| \phi_{j}(\mathbf{r})\right\rangle a_{i}^{\dagger} a_{j},
$$

where $\left|\phi_{i}(\mathbf{r})\right\rangle$ is the single-particle state identified by index $j_{z}$ in the LLL [21]. In our case, we also have an additional index to denote up- and down-spin components. Since $J_{z}$ is a good quantum number, the operator $a_{i}^{\dagger} a_{j}$ selects only one single-particle state within the LLL approximation. As a consequence, it does not contain information about products of different amplitudes and loses information about the interference pattern [21]. Hence, the density distribution solely preserves the information on individual densities,

$$
n(\mathbf{r})=\left\langle\Psi_{G}|\hat{\rho}(\mathbf{r})| \Psi_{G}\right\rangle=\sum_{i=1}^{M}\left|\phi_{i}(\mathbf{r})\right|^{2} O_{i},
$$

where $O_{i}$ is the total ground-state occupation of the singleparticle state $\left|\phi_{i}(\mathbf{r})\right\rangle$ [21]. Within the LLL approximation, $O_{i}$ are essentially eigenvalues of the diagonal single-particle density matrix. Since single-particle states in Eq. (4) are eigenstates of the $\mathcal{P}$ operator, it is evident that the density distributions $n(\mathbf{r})$ would be cylindrically symmetric. For example, representative plots of $O_{i}$ as a function of $j_{z}$ and plots of density distributions are shown in Figs. 6 and 9.

\section{Pair-correlation function}

Pair-correlation functions help us analyze the internal structure of the ground states. We write the pair-correlation operator (not normalized) in second-quantized form [21],

$$
\hat{\rho}\left(\mathbf{r}, \mathbf{r}_{0}\right)=\sum_{i j k l} \phi_{i}^{*}(\mathbf{r}) \phi_{j}^{*}\left(\mathbf{r}_{0}\right) \phi_{k}(\mathbf{r}) \phi_{l}\left(\mathbf{r}_{0}\right) a_{i}^{\dagger} a_{j}^{\dagger} a_{l} a_{k} .
$$

In our case, we also have an additional index to denote up- and down-spin components. For instance, we may compute paircorrelation functions that determine the conditional probability to find an up spin or a down spin, when an up-spin component is assumed to be present at a fixed point $\mathbf{r}_{0}$, i.e., $\left\langle n_{\uparrow}\left(\mathbf{r}_{0}\right) n_{\uparrow}(\mathbf{r})\right\rangle$ or $\left\langle n_{\uparrow}\left(\mathbf{r}_{0}\right) n_{\downarrow}(\mathbf{r})\right\rangle$, respectively. We may choose $\mathbf{r}_{0}$ to be away from the origin, but with a substantial amplitude of $n(\mathbf{r})$. Due to angular momentum conservation, the condition $i+j=k+l$ must further be fulfilled. By computing the expectation value of $\hat{\rho}\left(\mathbf{r}, \mathbf{r}_{0}\right)$ with respect to $\Psi_{G}$, we obtain the pair-correlation function as

$$
\begin{aligned}
\rho\left(\mathbf{r}, \mathbf{r}_{0}\right)= & \sum_{i j k l} \sum_{p p^{\prime}} \alpha_{p}^{*} \alpha_{p^{\prime}} \phi_{i}^{*}(\mathbf{r}) \phi_{j}^{*}\left(\mathbf{r}_{0}\right) \phi_{k}(\mathbf{r}) \phi_{l}\left(\mathbf{r}_{0}\right) \\
& \times\left\langle\Phi_{p}\left|a_{i}^{\dagger} a_{j}^{\dagger} a_{l} a_{k}\right| \Phi_{p^{\prime}}\right\rangle
\end{aligned}
$$

When the wave function $\Psi_{G}$ is an eigenstate of the $\mathcal{P} \mathcal{T}$ operator, the pair-correlation functions illustrate the ground-state symmetry properties. Furthermore, they reveal the correlations between up- and down-spin components in real space. Paircorrelation functions at representative interaction strengths are shown in Figs. 6 and 9.

\section{Reduced wave function}

We shall now discuss techniques to analyze whether the ground states possess vortex structures with distinct topological properties. One identifying property is the presence of quantized values of Skyrmion number, as discussed in our earlier work [18]. However, this requires the computation of the ground-state wave function in real space, a computationally prohibitive task for the bosonic few-particle system under study. Here, we discuss a viable approach to identify the topological nature of the ground state by computing the reduced wave function [22]:

$$
\psi_{r w f}(\mathbf{r})=\frac{\Psi\left(\mathbf{r}, \mathbf{r}_{2}^{*}, \ldots, \mathbf{r}_{\mathbf{N}}^{*}\right)}{\Psi\left(\mathbf{r}_{\mathbf{1}}^{*}, \mathbf{r}_{2}^{*}, \ldots, \mathbf{r}_{\mathbf{N}}^{*}\right)}
$$

Reduced wave function $\psi_{r w f}(\mathbf{r})$ is computed with respect to one particle, i.e., here the particle with index 1, while the remaining $N-1$ particles are placed at their most probable locations $\mathbf{r}_{\mathbf{i}}^{*}$ [22]. In our case, we also have an additional index to denote up- and down-spin components. With $\psi_{r w f, \uparrow}(\mathbf{r})$ and $\psi_{r w f, \downarrow}(\mathbf{r})$ known, we can now extract phase information and compute a distinct topological quantity, vorticity, i.e., the number of phase slips from $+\pi$ to $-\pi$ along a closed contour. An integer-valued vorticity is an unambiguous way 
of establishing that the ground state is topological in nature with a distinct vortex structure. For example, typical phase plots revealing different vorticities are shown in Figs. 6 and 9.

\section{Entanglement measures}

We compute entanglement measures to analyze correlation properties of various ground states. In particular, we intend to probe the ground-state correlation properties that specifically stem from the presence of interparticle interactions. To achieve this goal, we take cues from seminal papers in Ref. [23]. We choose a proper single-particle basis comprised of the set of eigenstates in Eq. (4) of the single-particle Hamiltonian $\mathcal{H}_{0}$. In such a single-particle basis, entanglement in the ground state, or any nondegenerate energy eigenstate, occurs specifically due to the presence of interactions [23].

The first step in discussing any entanglement measure is to partition the system and compute entanglement properties between different subsystems. As discussed in Sec. IIC, similar to 2D LL orbitals, the single-particle eigenstates at large SO-coupling strengths are fairly localized in nature. This warrants us to consider partitioning the system in orbital space [24]. The $\mathcal{T}$ symmetry preserved by the Hamiltonian $\mathcal{H}$ naturally prompts us to partition the orbitals into two subsystems: positive $j_{z}$ states (subsystem $A$ ) and negative $j_{z}$ states (subsystem $B$ ). We write the ground-state wave function in Fock space as $\Psi_{G}=\sum_{p=1}^{n_{d}} \alpha_{p} \Phi_{p}$, where $\Phi_{p}$ is represented as $\left|n_{-j_{c}} n_{-\left(j_{c}-1\right)} \cdots n_{j_{c}-1} n_{j_{c}}\right\rangle$. Here, $n_{j_{z}}$ represents the occupation number of the single-particle eigenstate $j_{z}$, and, as discussed in Sec. IID, a finite-size cutoff is made at a certain value $j_{c} \equiv j_{z, c}$ for computational feasibility. Now, we proceed to compute the bipartite entanglement properties between subsystems $A$ and $B$, i.e., between the positive and negative $j_{z}$ states, respectively.

Orbital entanglement spectrum. With the knowledge of $\Psi_{G}$, we compute the entries of the density matrix $\hat{\rho}$ for the ground state as

$$
\left\langle n_{-j_{c}}^{\prime} \cdots n_{j_{c}}^{\prime}|\hat{\rho}| n_{-j_{c}} \cdots n_{j_{c}}\right\rangle=\alpha_{p} \alpha_{p}^{*},
$$

where the generic density operator is $\hat{\rho}=\left|\Psi_{G}\right\rangle\left\langle\Psi_{G}\right|$.

Now, we compute the reduced density matrix (RDM) $\hat{\rho}_{A}$ by tracing out the degrees of freedom of subsystem $B$, meaning $\hat{\rho}_{A}=\operatorname{Tr}_{B} \hat{\rho}$. As shown in Ref. [23], occupation numbers act as distinguishable degrees of freedom in characterizing entanglement in a finite system of identical quantum particles. Hence, in our study, RDM is computed by tracing out the occupation of all the negative $j_{z}$ states from the density matrix,

$$
\begin{aligned}
& \left\langle n_{1 / 2}^{\prime} \cdots n_{j_{c}}^{\prime}\left|\hat{\rho}_{j_{c}}\left(1 / 2, \ldots, j_{c}\right)\right| n_{1 / 2} \cdots n_{j_{c}}\right\rangle \\
& =\sum_{n_{-j_{c}} \cdots n_{-1 / 2}}\left\langle n_{-j_{c}} \cdots n_{-1 / 2} n_{1 / 2}^{\prime} \cdots n_{j_{c}}^{\prime}\right| \\
& \quad \times \hat{\rho}\left|n_{-j_{c}} \cdots n_{-1 / 2} n_{1 / 2} \cdots n_{j_{c}}\right\rangle .
\end{aligned}
$$

The RDM $\hat{\rho}_{A}$ has a block-diagonal structure, with each block characterized by the total angular momentum $J_{z}^{A}$ that corresponds only to particles in subsystem $A$. The block-diagonal structure allows us to compute all the eigenvalues of the RDM using full-diagonalization techniques. Orbital entanglement spectrum (OES), termed so because the partition is defined in orbital space, is the plot of entanglement pseudoenergies $\xi_{i}$ as a function of $J_{z}^{A}$. Here, $\xi_{i}=-\ln \rho_{i}^{A}$, with $\rho_{i}^{A}$ being the eigenvalues of RDM $\hat{\rho}_{A}$ [25]. It is evident that $\xi_{i}$ with smaller magnitudes maximally contribute to the ground-state properties.

Plots of OES reveal information about the occupation of various Fock states in a given ground-state manifold and, in turn, the correlation properties of the ground state. If various Fock states $\Phi_{p}$ in the ground-state $J_{z}$ manifold have similar magnitudes of $\alpha_{p}$, then it results in similar RDM eigenvalues of $\rho_{i}^{A}$ and, in turn, similar magnitudes of $\xi_{i}$. Thus, if an OES plot reveals that $\xi_{i}$ values are degenerate or nearly degenerate, then this is a clear manifestation of the correlated nature of the ground state. On the other hand, if the OES plot reveals that the values of $\xi_{i}$ are distinctly nondegenerate, then the ground state is clearly not correlated. For example, representative OES plots are shown in Figs. 6, and 9.

Entanglement entropy. Plots of OES reveal the whole spectrum of eigenvalues of the RDM and help us understand the correlation properties of the ground state. However, it is sometimes useful to extract just a single representative quantity from the RDM [26]. Entanglement entropy (EE) is such a measure that can be readily obtained from the set of eigenvalues $\rho_{i}^{A}$ of the $\operatorname{RDM} \hat{\rho}_{A}$, and is defined as $S_{A}=-\operatorname{tr}\left[\hat{\rho}_{A} \ln \hat{\rho}_{A}\right]=-\sum_{i} \rho_{i}^{A} \ln \rho_{i}^{A}$. A higher entropy value means that the ground state is more homogeneously spread in Fock space, i.e., a larger number of Fock states $\Phi_{p}$ make substantial contributions towards the ground state. A distinct advantage of an EE plot is that we are able to look at entropy values for a whole range of interaction strengths in a single plot, and thereby understand correlation properties of various phases. For example, representative EE plots are shown in Figs. 4, 5, 7, and 8.

In summary, density distribution, eigenvalues of the singleparticle density matrix, pair-correlation function, and reduced wave function would help us identify various symmetry and topological properties of the ground states. The computation of RDM from a proper single-particle basis enables us to extract various entanglement measures and allows us to analyze

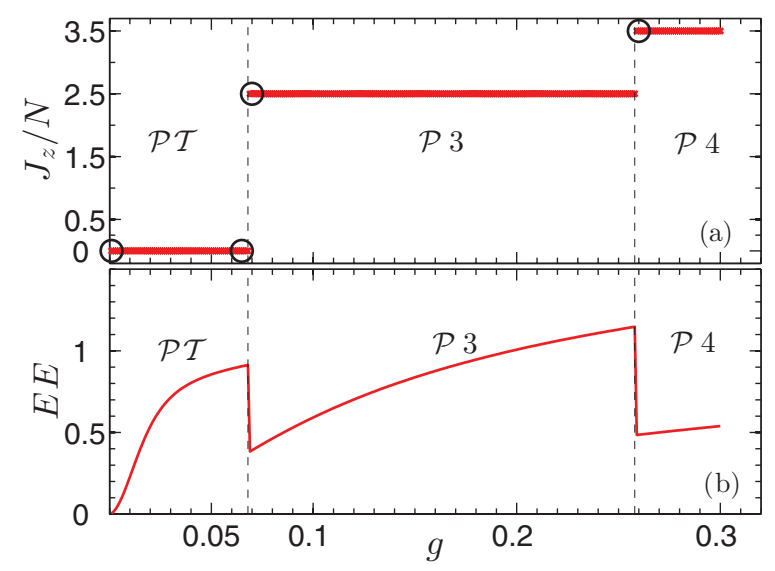

FIG. 4. (Color online) Plots of (a) ground-state $J_{z} / N$ manifolds and (b) entanglement entropy, as a function of interaction strength $g$ with $\lambda_{\text {SO }}=20, N=2, g_{\uparrow \downarrow} / g=0.5$. For representative interaction strengths, denoted by circled (black) markers, we illustrate the ground-state properties in Fig. 6. 


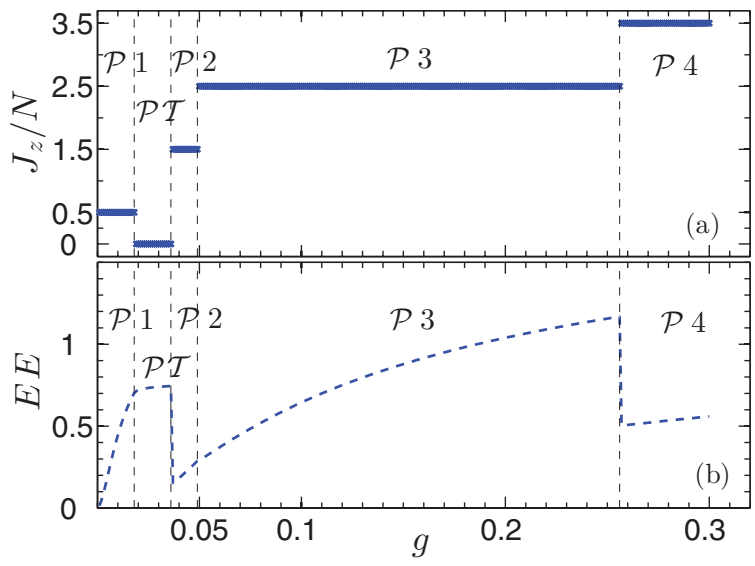

FIG. 5. (Color online) Plots of (a) ground-state $J_{z} / N$ manifolds and (b) entanglement entropy, as a function of interaction strength $g$ with $\lambda_{\text {SO }}=20, N=2, g_{\uparrow \downarrow} / g=1.5$.

correlation properties that specifically stem from interparticle interactions.

\section{RESULTS AND DISCUSSION}

As discussed in Sec. II C, in the absence of interactions, all particles would simply condense into the two lowest-energy single-particle eigenstates in the LLL identified by quantum numbers $j_{z}= \pm 0.5$. This is due to the weak, but finite, dispersion in $j_{z}$ present in the single-particle energy spectrum shown in Fig. 2(b). The $\mathcal{P}$ eigenstate, identified by $j_{z}=+0.5$, is represented by wave function $\Phi_{\mathcal{P}}=\left[\phi_{\uparrow}(\rho), \phi_{\downarrow}(\rho) e^{i \varphi}\right]^{T} / \sqrt{2 \pi}$. It has a half-quantum vortex configuration, as the spin-up component stays in the $s$ state and the spin-down component is in the $p$ state $[18,27,28]$. The resulting spin texture of this topological state is of the Skyrmion type [18]. The degenerate time-reversed $\mathcal{P}$ eigenstate, identified by $j_{z}=-0.5$ and represented by $\mathcal{T} \Phi_{\mathcal{P}}=\left[\phi_{\downarrow}(\rho) e^{-i \varphi},-\phi_{\uparrow}(\rho)\right]^{T} / \sqrt{2 \pi}$, also has a half-quantum vortex configuration. We may as well construct a zero angular momentum $\mathcal{P} \mathcal{T}$ eigenstate from an equal superposition of opposite angular momentum $\mathcal{P}$ eigenstates: $\Phi_{\mathcal{P} \mathcal{T}, j_{z}=0}=\left(\Phi_{\mathcal{P}} \pm \mathcal{T} \Phi_{\mathcal{P}}\right) / \sqrt{2}$. In the absence of interactions, either of the $\mathcal{P}$ eigenstates or the superposition $\mathcal{P} \mathcal{T}$ eigenstate are degenerate. In addition, any arbitrary superposition of the degenerate $\mathcal{P}$ eigenstates, which, in principle, need not be a $\mathcal{P} \mathcal{T}$ eigenstate, will also be a degenerate ground state.

In the presence of interparticle interactions, the ground state is no longer determined solely by the energy contribution of the noninteracting part of the Hamiltonian $\mathcal{H}_{0}$. Depending upon the strengths of $g$ and $g_{\uparrow \downarrow}$, the energy contribution from the interacting part of the Hamiltonian $\mathcal{H}_{\text {int }}$ also plays a crucial role. This competition can be better understood, especially at large SO-coupling strengths, by analyzing the single-particle wave functions and energy. As shown in Fig. 2(b), energy contributions due to $\mathcal{H}_{0}$ try to keep the particles in states with a lower value of angular momenta $j_{z}$. However, for repulsive interaction strengths, energy considerations due to $\mathcal{H}_{\text {int }}$ try to keep the particles as far away from each other as possible. This, in turn, means that the particles tend to occupy states with a larger value of angular momenta, since they have a larger localization radii, as shown in Fig. 1(b). In essence, the ground state of the interacting many-body Hamiltonian is determined by the competition between the $\mathcal{H}_{0}$ and $\mathcal{H}_{\text {int }}$ terms.

The simplest scenario where the competition between the $\mathcal{H}_{0}$ and $\mathcal{H}_{\text {int }}$ terms and, in turn, the effect of interparticle interactions clearly manifests is in an interacting problem with $N=2$ particles. For this reason, we discuss the results for $N=2$ particles and analyze the ground-state properties in greater detail before proceeding to larger particle numbers. We solve the interacting few-body Hamiltonian $\mathcal{H}$ at large SO-coupling strengths using the ED scheme within the LLL approximation. The computational intensity, especially at large interaction strengths, limits the feasibility of this scheme to the order of $N=8$ particles. In an earlier mean-field study on homogeneous two-component Bose gas [29], it was shown that the particles condense into either a single plane-wave state (for $g>g_{\uparrow \downarrow}$ ) or a density-stripe state (for $g<g_{\uparrow \downarrow}$ ). Similarly, in our earlier related work on trapped two-component Bose gas [30], depending on the relative magnitudes of $g$ and $g_{\uparrow \downarrow}$, we show that states with distinct topological and symmetry properties emerge in the mean-field phase diagram. Taking cues from these results, in this study, we solve for the groundstate wave function at various interaction strengths; however, we fix the relative magnitude $g_{\uparrow \downarrow} / g$ at 0.5 or 1.5 . In this section, we present the results at different particle numbers $N$, and analyze the topological, symmetry, and correlation properties of the ground states using various techniques discussed in Sec. II E.

\section{A. $N=2$}

As discussed in Sec. II E1, we analyze the energy spectrum to identify the ground-state angular momentum manifold $J_{z}$ or, equivalently, $J_{z} / N$. In Fig. 3(a), we notice that the ground state belongs to the $J_{z} / N=0$ manifold. We further determine that the ground-state wave function $\Psi_{G}$ is an eigenstate of the $\mathcal{P T}$ operator. On the other hand, we observe from Fig. 3(b) that the ground state is degenerate in $J_{z} / N= \pm 0.5$ manifolds. In either scenario, in Fig. 3(b), we determine that $\Psi_{G}$ is an eigenstate of the $\mathcal{P}$ operator. It is evident that even in the presence of extremely weak-interaction strengths, the interacting Hamiltonian picks either a $\mathcal{P}$ eigenstate or a $\mathcal{P} \mathcal{T}$ eigenstate to be the ground state. Furthermore, it is clear that the ground state is sensitive to the relative magnitudes of $g_{\uparrow \downarrow}$ and $g$.

Figures 4(a) and 5(a). We solve the interacting Hamiltonian $\mathcal{H}$ at various interaction strengths and identify corresponding ground-state manifolds $J_{z} / N$ in Figs. 4(a) and 5(a). It is evident from the phase diagram that depending on $g$ and $g_{\uparrow \downarrow}$, the ground states belong to different $J_{z} / N$ manifolds. Furthermore, we determine if the ground-state wave function $\Psi_{G}$ is an eigenstate of the $\mathcal{P} \mathcal{T}$ operator, and thereby identify whether the state belongs to the $\mathcal{P}$ or $\mathcal{P} \mathcal{T}$ symmetry phase. In a broader sense, it is evident that a ground state in the $\mathcal{P} \mathcal{T}$ symmetry phase belongs to the $J_{z} / N=0$ manifold, while ground states in various $J_{z} / N \neq 0$ manifolds belong to the $\mathcal{P}$ symmetry phase. EE plots in Figs. 4(b) and 5(b) reveal correlation properties in various phases. For pedagogical purposes, before we explain the features in EE plots, we first discuss the symmetry, topological, and correlation properties of ground states. 

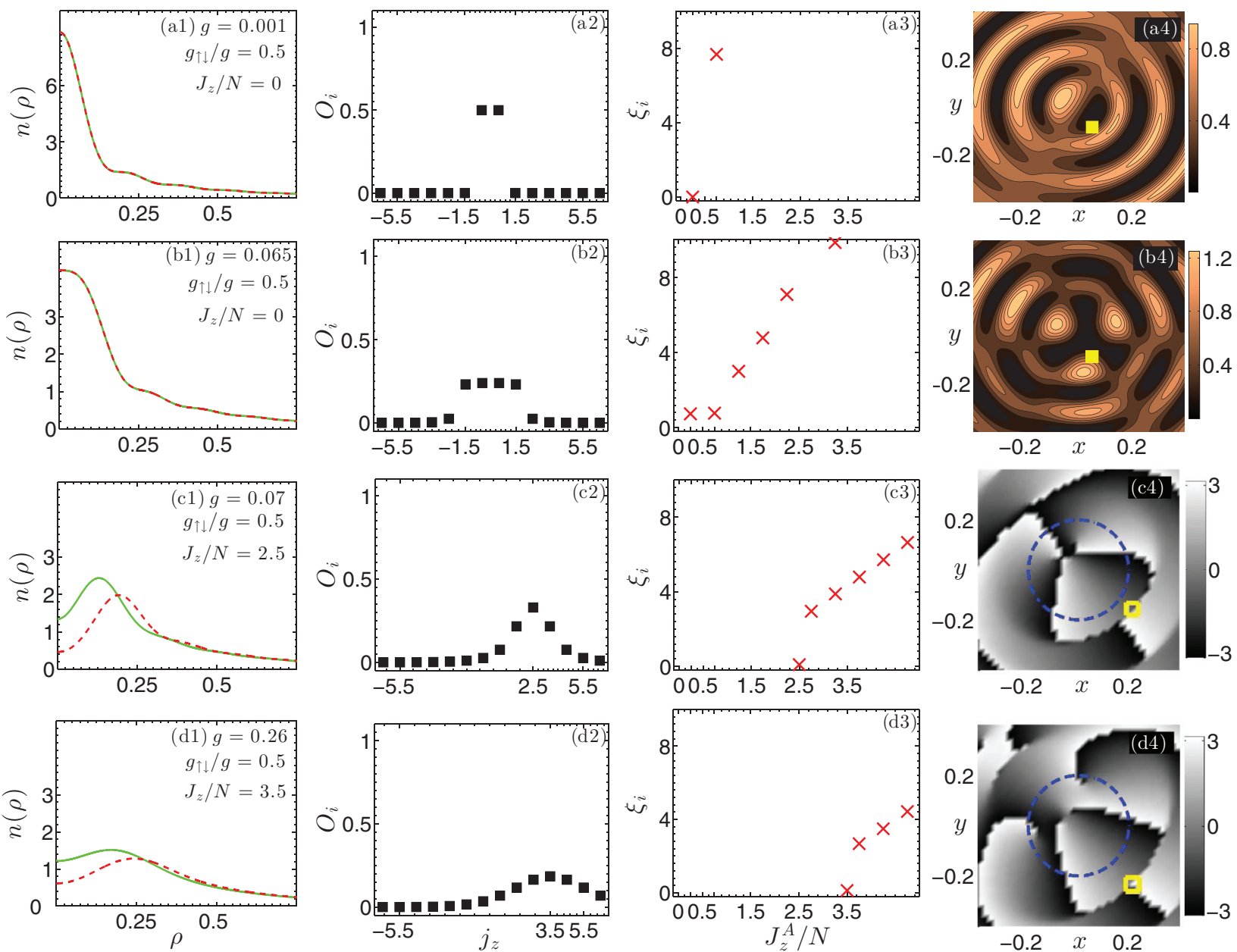

FIG. 6. (Color online) Plots in each row illustrate the ground-state properties at a representative interaction strength of Fig. 4(a). In the first column (from left), we show density distributions of spin-up component $n_{\uparrow}(\rho)$ (solid green line) and of spin-down component $n_{\downarrow}(\rho)($ dashed red line). In the second column, we show eigenvalues $O_{i}$ of the single-particle density matrix as a function of angular momentum $j_{z}$ of the single-particle states $\left|\phi_{i}(\mathbf{r})\right\rangle$. In the third column, we show corresponding OES plots of entanglement pseudoenergies $\xi_{i}$ as a function of $J_{z}^{A} / N$, the average angular momentum of subsystem $A$. In the last column, we show contour plots (a4) and (b4) that are normalized pair-correlation functions $\left\langle n_{\uparrow}\left(\mathbf{r}_{0}\right) n_{\downarrow}(\mathbf{r})\right\rangle$, with $\mathbf{r}_{0}$ denoted by a (yellow) marker. Phase plots (c4) and (d4) are derived from reduced wave function $\psi_{c, \downarrow}(\mathbf{r})$, which is computed by fixing one of the two particles at their most probable locations, and their corresponding radii are indicated by (yellow) markers. The closed dashed (blue) contour is a guide to the eye, which allows us to count the number of phase slips.

In Fig. 6, we illustrate density distributions, eigenvalues of the single-particle density matrix, orbital entanglement spectrum, pair-correlation functions, and reduced wave functions at representative interaction strengths within various $J_{z} / N$ manifolds of Fig. 4(a). Using a similar line of reasoning, we may understand the properties of ground states in Fig. 4(b). Let us now proceed to discuss various plots shown in Fig. 6.

Figures 6(al)-6(a4). In this top row, we discuss the ground-state properties of the $\mathcal{P} \mathcal{T}$ eigenstate in the $J_{z} / N=0$ manifold at $g=0.001$ of Fig. 4(a). As shown in Fig. 6(a1), the cylindrically symmetric density distributions $n_{\uparrow}(\rho)$ and $n_{\downarrow}(\rho)$ overlap. Being a $\mathcal{P} \mathcal{T}$ eigenstate, it is evident from Fig. 6(a2) that the positive and negative angular momentum states are equally occupied. Furthermore, the time-reversal partner states identified by quantum numbers $j_{z}= \pm 0.5$ are predominantly occupied. As expected, from the corresponding OES plot in Fig. 6(a3), we observe that the predominant contribution to the ground state is from the entanglement pseudoenergy $\xi_{i}$ at $J_{z}^{A} / N=+0.25$. From Figs. 6(a2) and 6(a3), it is clear that the the maximally contributing Fock state is $\Phi_{\mathcal{P T}}=$ $\left|n_{j_{z}=-0.5}=1, n_{j_{z}=+0.5}=1\right\rangle$, which explains the overlapping density distributions of $n_{\uparrow}(\rho)$ and $n_{\downarrow}(\rho)$ in Fig. 6(a1). In Fig. 6(a4), we plot the (normalized) pair-correlation function $\left\langle n_{\uparrow}\left(\mathbf{r}_{0}\right) n_{\downarrow}(\mathbf{r})\right\rangle$ of this $\mathcal{P} \mathcal{T}$ eigenstate. This plot illustrated the conditional probability to find a down spin, when an up-spin component is assumed to be at a fixed point $\mathbf{r}_{0}$, and reveals the presence of correlated regions (magnitude closer to 1) and anticorrelated regions (magnitude closer to 0 ). This plot illustrates the correlations present between up-spin and downspin components that are not revealed by the cylindrically symmetric density distributions.

Figures $6(b 1)-6(b 4)$. In this second row, we discuss the ground-state properties of the $\mathcal{P} \mathcal{T}$ eigenstate in the $J_{z} / N=0$ manifold at $g=0.065$ of Fig. 4(a). As discussed with reference to Fig. 6(a1), the density distributions $n_{\uparrow}(\rho)$ and $n_{\downarrow}(\rho)$ overlap in Fig. 6(b1). It is evident from Fig. 6(b2) that the time-reversal 
partner states identified by $j_{z}= \pm 0.5$ and $j_{z}= \pm 1.5$ are almost equally occupied. From the corresponding OES plot in Fig. 6(b3), we observe that the ground state is equally occupied by $\xi_{i}$ at $J_{z}^{A} / N=+0.25$ and +0.75 . From Figs. $6(\mathrm{~b} 2)$ and 6(b3), it is clear that the the maximally contributing Fock states are $\Phi_{\mathcal{P} \mathcal{T}}=\left|n_{j_{z}=-0.5}=1, n_{j_{z}=+0.5}=1\right\rangle$ and $\Phi_{\mathcal{P} \mathcal{T}}=$ $\left|n_{j_{z}=-1.5}=1, n_{j_{z}=+1.5}=1\right\rangle$. To illustrate the internal structure of this $\mathcal{P} \mathcal{T}$ eigenstate and the correlations between up-spin and down-spin components, we show the pair-correlation function in Fig. 6(b4).

Figures 6(c1)-6(c4). In this third row, we discuss the ground-state properties of the $\mathcal{P}$ eigenstate in the $J_{z} / N=$ +2.5 manifold at $g=0.07$ of Fig. 4(a). While the corresponding ground state is degenerate in $J_{z} / N= \pm 2.5$ manifolds, we restrict our discussion to the $J_{z} / N=+2.5$ manifold without loss of generality. The cylindrically symmetric density distributions $n_{\uparrow}(\rho)$ and $n_{\downarrow}(\rho)$ are distinct, as shown in Fig. 6(c1). In this $\mathcal{P}$ eigenstate, there is an inherent asymmetry in the occupation of positive and negative angular momentum states. This is evident from the plot of single-particle density-matrix eigenvalues $O_{i}$ in Fig. 6(c2). This explains the presence of distinct density distributions in Fig. 6(c1). Furthermore, we observe a peak in the occupation of the eigenstate identified by $j_{z}=+2.5$ in Fig. 6(c2). From the corresponding OES plot in Fig. 6(c3), we observe that the ground state is predominantly occupied by $\xi_{i}$ at $J_{z}^{A} / N=2.5$. To illustrate the internal structure of this $\mathcal{P}$ eigenstate, we show the phase plot derived from the reduced wave function $\psi_{c, \downarrow}(\mathbf{r})$ in Fig. 6(c4). To better understand this phase plot, we take cues from plots in Figs. 6(c2) and 6(c3). Though we observe from Fig. 6(c3) that the ground state is predominantly occupied by $\xi_{i}$ at $J_{z}^{A} / N=$ 2.5, it may be conceived from Fig. 6(c2) that the ground state has contributions from various Fock states; for example, $\Phi_{\mathcal{P}}=\left|n_{j_{z}=+2.5}=2\right\rangle$ or $\Phi_{\mathcal{P}}=\left|n_{j_{z}=+1.5}=1, n_{j_{z}=+3.5}=1\right\rangle$ or $\Phi_{\mathcal{P}}=\left|n_{j_{z}=+0.5}=1, n_{j_{z}=+4.5}=1\right\rangle$. From the representation of single-particle eigenstates in Eq. (4), it is evident that the net orbital angular momentum of the spin-up component in the ground state is +2 and that of the spin-down component is +3 . Correspondingly, the phase plot of the down-spin component in Fig. 6(c4) reveals a vorticity of 3 . We note here that the vorticity is the number of phase slips from $+\pi$ to $-\pi$, i.e., when the shadowing changes from white to black. For convenience, we identify this $\mathcal{P}$ eigenstate as $\mathcal{P} 3$, where 3 is the vorticity of the down-spin component.

Figures $6(d 1)-6(d 4)$. In this last row, we discuss the ground-state properties of the $\mathcal{P}$ eigenstate in the $J_{z} / N=$ +3.5 manifold at $g=0.26$ of Fig. 4(a). The corresponding ground state is degenerate in $J_{z} / N= \pm 3.5$ manifolds, while we restrict our discussion to the $J_{z} / N=+3.5$ manifold. As expected for a $\mathcal{P}$ eigenstate, the density distributions $n_{\uparrow}(\rho)$ and $n_{\downarrow}(\rho)$ shown in Fig. 6(d1) are distinct. In addition to the asymmetric occupation of positive and negative angular momentum states in Fig. 6(d2), we observe a peak occupation of the eigenstate identified by $j_{z}=+3.5$. From the corresponding OES plot in Fig. 6(d3), we observe that the ground state is predominantly occupied by $\xi_{i}$ at $J_{z}^{A} / N=3.5$. However, it may be conceived from Fig. 6(d2) that the ground state has contributions from various Fock states; for example, $\Phi_{\mathcal{P}}=1$ $\left.n_{j_{z}=+3.5}=2\right\rangle$ or $\Phi_{\mathcal{P}}=\left|n_{j_{z}=+1.5}=1, n_{j_{z}=+5.5}=1\right\rangle$ or $\Phi_{\mathcal{P}}=\mid$ $\left.n_{j_{z}=+2.5}=1, n_{j_{z}=+4.5}=1\right\rangle$. It is clear that with increasing interparticle interaction strengths, the particles distribute themselves in higher angular momentum manifolds. Furthermore, it is evident that the net orbital angular momentum of the spin-up component in the ground state is +3 and that of the spin-down component is +4 . Correspondingly, the phase plot of the down-spin component in Fig. 6(d4) reveals a vorticity of 4. For convenience, we identify this $\mathcal{P}$ eigenstate as $\mathcal{P} 4$. We further note that the phase plots of the down-spin components derived for $\mathcal{P} 1$ and $\mathcal{P} 2$ eigenstates in Fig. 5(a) exhibit a vorticity of 1 and 2 , respectively.

Figures 4(b) and 5(b). As noted earlier, OES preserves the whole spectrum of eigenvalues of the RDM, and hence allows us to extract information about the occupation of Fock states with different subsystem angular momenta $J_{z}^{A}$. With our understanding of the OES plots in Figs. 6, we now proceed to explain various features observed in the EE plots of Figs. 4(b) and 5(b): (i) The presence of distinctly different slopes suggests the presence of distinct correlation properties in ground states within various phases. (ii) Within each phase, EE increases monotonously with increasing $g$. As discussed in Sec. IIE5, this results from an increasingly homogeneous distribution of Fock states in the ground-state $J_{z} / N$ manifold and, in turn, an increased correlation. For example, to illustrate this feature within the $\mathcal{P} \mathcal{T}$-symmetric phase in Fig. 4(b), we may compare the OES plots in Figs. 6(a3) and 6(b3) and observe an increased homogeneity in the distribution of Fock states. (iii) The presence of nearly degenerate $\xi_{i}$ values results in a reduction in the slope of $\mathrm{EE}$. While this feature is observed at larger interaction strengths within the $\mathcal{P} \mathcal{T}$-symmetric phase of Fig. 4(b), the OES plot in Fig. 6(b3) helps us understand this. (iv) The transition to a $\mathcal{P}$-symmetric phase is marked by a sharp reduction in the value of EE [31]. To better understand this feature, we compare the OES plots in Figs. 6(b3) and 6(c3) and observe a sharp reduction in homogeneity of the $\xi_{i}$ values, accompanied by a substantial drop in the minimum value of $\xi_{i}$. In summary, we emphasize that knowledge of the OES helps us understand various features exhibited by the EE plots.

In summary, it is evident that the interacting Hamiltonian picks either a $\mathcal{P}$ eigenstate or a $\mathcal{P} \mathcal{T}$ eigenstate to be the ground state. The ground state is sensitive to the relative magnitudes of $g_{\uparrow \downarrow}$ and $g . J_{z} / N$ plots allow us to identify various $\mathcal{P}$ and $\mathcal{P} \mathcal{T}$ symmetry phases in the interacting system. With the analysis of density distributions, the single-particle density matrix, and reduced wave functions, we illustrate ground-state symmetry and topological properties. We assert that the bosons condense into an array of $\mathcal{P}$-symmetric topological ground states that have $n+1 / 2$ quantum angular momentum vortex configuration, with $n=0,1,2,3$. With the analysis of the single-particle density matrix, OES, and pair-correlation functions, we illustrate the internal structure of different ground states in the $\mathcal{P} \mathcal{T}$ symmetry phase. We analyze the correlation properties of the ground states with the help of the OES and EE plots.

\section{B. $N=8$}

The detailed analysis presented above for the relatively simple, but rich, scenario of $N=2$ particles shall be useful when discussing results at larger particle numbers. Even with a small increase in particle number from $N=2$ to $N=4$ 


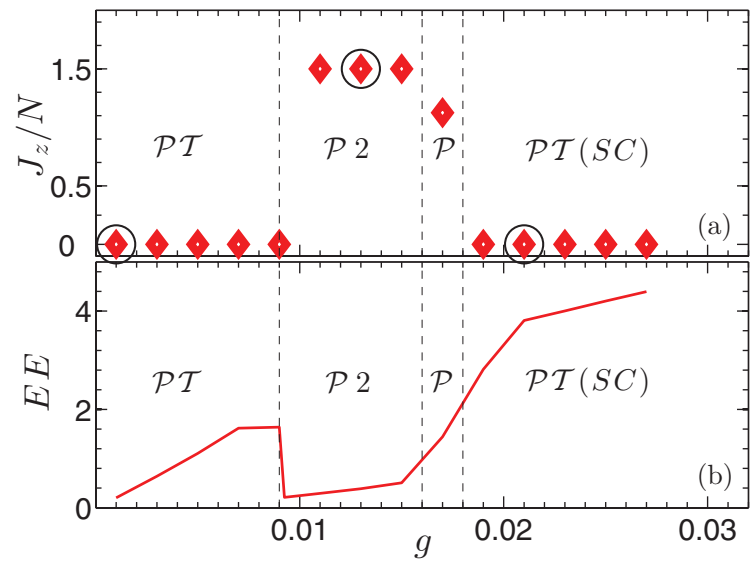

FIG. 7. (Color online) Plots of (a) ground state $J_{z} / N$ manifolds and (b) entanglement entropy, as a function of interaction strength $g$ with $\lambda_{\text {SO }}=20, N=8, g_{\uparrow \downarrow} / g=0.5$. For representative interaction strengths denoted by circled (black) markers, we illustrate the groundstate properties in Fig. 9.

(not shown), we observe the nonoccurrence of ground states in the $\mathcal{P} 4$ phase. As discussed in the introduction of Sec. III, this can be understood as a manifestation of the competition between energy contributions from $\mathcal{H}_{0}$ and $\mathcal{H}_{\text {int }}$. A higher particle number increases the probability distribution into single-particle states with smaller angular momenta, when compared to larger angular momenta eigenstates. We shall now proceed to consider the few-body system with $N=8$ particles, discuss the occurrence of various phases, and analyze the ground-state properties at representative interaction strengths using various techniques outlined in Sec. II E.

Figures $7(a)$ and $8(a)$. We solve the interacting Hamiltonian $\mathcal{H}$ at various interaction strengths and identify corresponding ground-state manifolds $J_{z} / N$ in Figs. 7(a) and 8(a). As discussed with reference to Figs. 4(a) and 5(a), it is evident that depending on $g$ and $g_{\uparrow \downarrow}$, the ground states belong to different $J_{z} / N$ manifolds and, in turn, to $\mathcal{P} \mathcal{T}$ or $\mathcal{P}$ symmetry phases. In this relatively larger particle number scenario, we

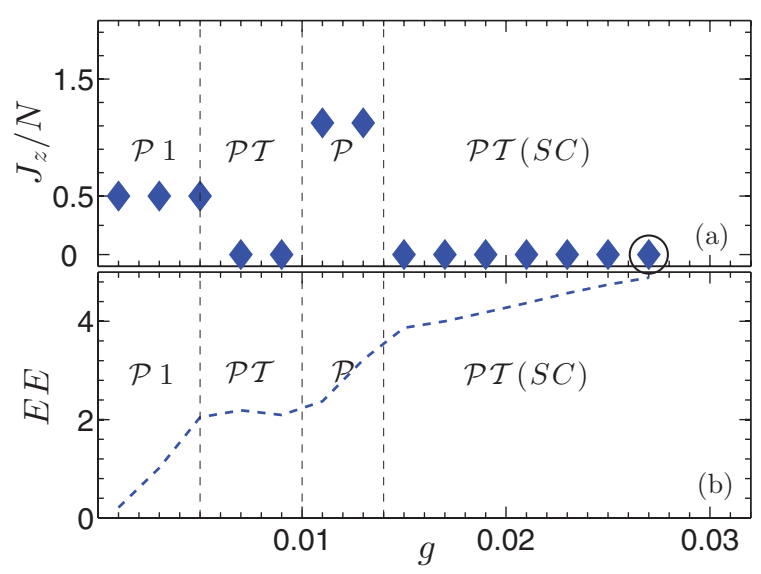

FIG. 8. (Color online) Plots of (a) ground state $J_{z} / N$ manifolds and (b) entanglement entropy, as a function of interaction strength $g$ with $\lambda_{\text {SO }}=20, N=8, g_{\uparrow \downarrow} / g=1.5$. For the representative interaction strength, denoted by a circled (black) marker, we illustrate the ground-state properties in Fig. 9. observe that the ground states fall into two distinct regimes: (a) at weak-interaction strengths (mean-field-like regime), we observe ground states with topological and symmetry properties that are consistent with mean-field theory computations [30]; (b) at intermediate- to strong-interaction strengths (strongly correlated regime), we report the emergence of strong correlations in ground states. The strongly correlated ground states are eigenstates of the $\mathcal{P} \mathcal{T}$ operator, and we additionally identify them with the label SC. In Fig. 9, we illustrate the ground-state properties at representative interaction strengths in these two regimes.

Mean-field-like regime: Figs. 9(al)-9(a4) and 9(b1)-9(b4). In the top row, we illustrate the ground-state properties of the $\mathcal{P} \mathcal{T}$ eigenstate in the $J_{z} / N=0$ manifold at $g=0.001$ of Fig. 7(a). It is evident that the properties in Figs. 9(a1)-9(a4) are qualitatively identical to their counterparts in Figs. 6(a1)6(a4). In the second row, we discuss the ground-state properties of the $\mathcal{P}$ eigenstate in the $J_{z} / N=+1.5$ manifold at $g=0.013$ of Fig. 7(a). The corresponding ground state is degenerate in $J_{z} / N= \pm 1.5$ manifolds, while we restrict our discussion to the $J_{z} / N=+1.5$ manifold. As expected for a $\mathcal{P}$ eigenstate, the density distributions $n_{\uparrow}(\rho)$ and $n_{\downarrow}(\rho)$ shown in Fig. 9(b1) are distinct. It is evident from the single-particle densitymatrix eigenvalues in Fig. 9(b2) that there is a peak in the occupation of the eigenstate identified by $j_{z}=+1.5$. From the corresponding OES plot in Fig. 9(b3), we observe that the ground state is predominantly occupied by $\xi_{i}$ at $J_{z}^{A} / N=1.5$. To illustrate the internal structure of this $\mathcal{P}$ eigenstate, we show the phase plot derived from the reduced wave function $\psi_{c, \downarrow}(\mathbf{r})$ in Fig. 9(b4). It is evident from the representation in Eq. (4) that the orbital angular momentum of the spin-up component in the ground state is +1 and that of the spin-down component is +2 . Correspondingly, the phase plot of the down-spin component shown in Fig. 9(b4) exhibits a vorticity of 2 , and hence we identify this $\mathcal{P}$ eigenstate as $\mathcal{P} 2$.

Strongly correlated regime: Figs. 9(cl)-9(c4) and 9(dl)$9(d 4)$. In the third and fourth rows, we illustrate the groundstate properties of the $\mathcal{P} \mathcal{T}$ eigenstates in the strongly correlated regime at $g=0.021$ of Fig. 7(a) and $g=0.027$ of Fig. 8(a), respectively. At intermediate- to strong-interaction strengths, as shown in Figs. 7(a) and Fig. 8(a), all of the ground states in this regime are eigenstates of the $\mathcal{P} \mathcal{T}$ operator in the $J_{z} / N=0$ manifold. As expected, the density distributions $n_{\uparrow}(\rho)$ and $n_{\downarrow}(\rho)$ overlap in Figs. 9(c1) and 9(d1). We observe that the density distributions become increasingly flat with increasing magnitude of interaction strengths, $g$ and $g_{\uparrow \downarrow}$. The interaction-induced correlations present in the ground states are revealed by the eigenvalues of the single-particle density matrix and OES plots. From the plots in Figs. 9(c2) and 9(d2), it is evident that the particles are nearly uniformly distributed across many single-particle eigenstates, with an equal distribution among time-reversal partner states. This distribution is qualitatively in the opposite limit to the corresponding plots in the mean-field-like regime illustrated in Figs. 9(a2) and 9(b2). This feature is further substantiated in the OES plots of Figs. $9(\mathrm{c} 3)$ and $9(\mathrm{~d} 3)$, where a large number of entanglement pseudoenergies $\xi_{i}$ are degenerate or nearly degenerate. As discussed in Sec. II E5, the presence of a large degeneracy in entanglement pseudoenergies is a clear manifestation of the strongly correlated nature of the ground states. We further 

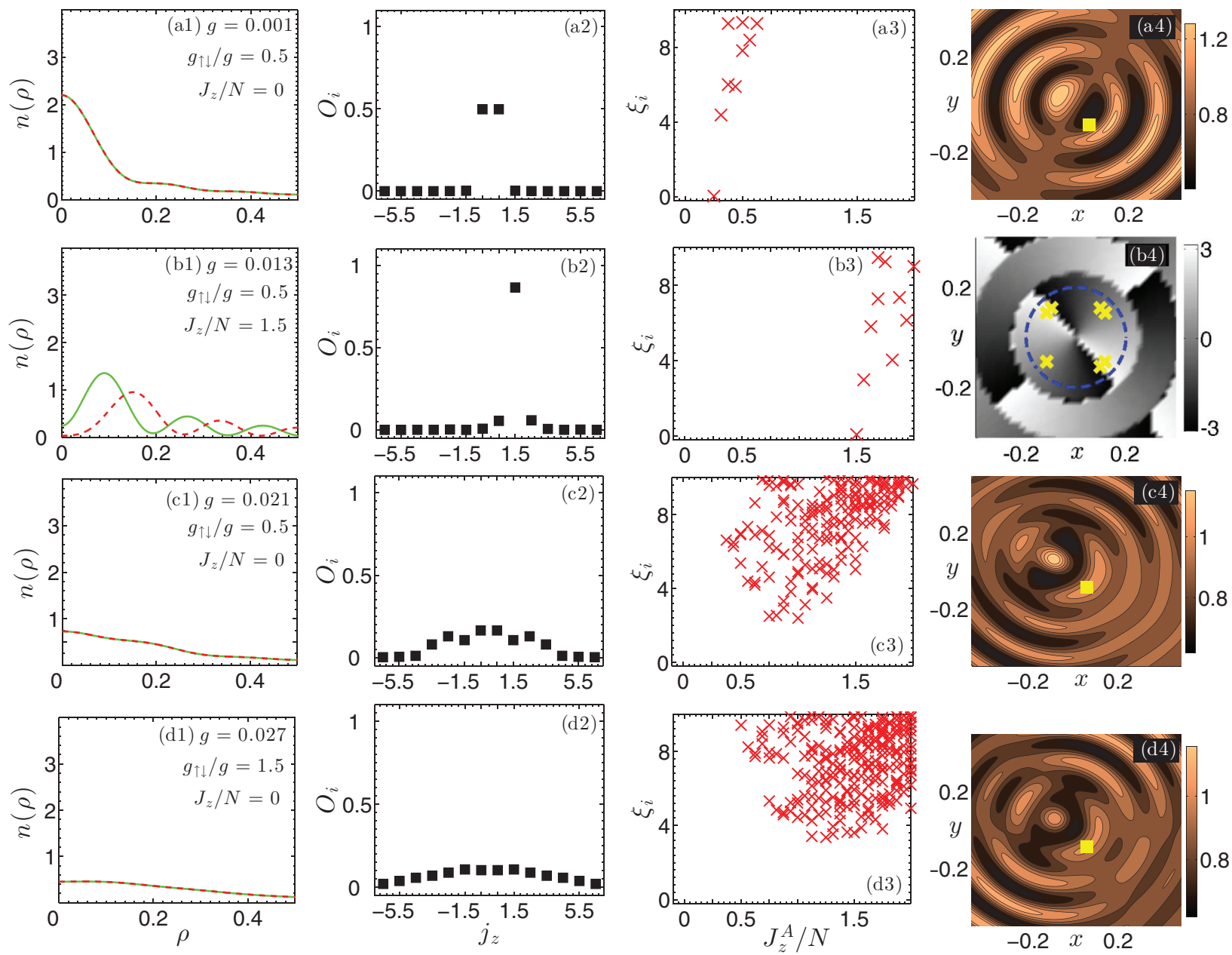

FIG. 9. (Color online) Plots in each row illustrate the ground-state properties at a representative interaction strength in Fig. 7(a) or 8(a). In the first column (from left), we show density distributions of the spin-up component $n_{\uparrow}(\rho)$ (solid green line) and of the spin-down component $n_{\downarrow}(\rho)$ (dashed red line). In the second column, we show eigenvalues $O_{i}$ of the single-particle density matrix as a function of angular momentum $j_{z}$ of the single-particle states $\left|\phi_{i}(\mathbf{r})\right\rangle$. In the third column, we show corresponding OES plots of entanglement pseudoenergies $\xi_{i}$ as a function of $J_{z}^{A} / N$, the average angular momentum of subsystem $A$. In the last column, we show contour plots (a4), (c4), and (d4) that are normalized pair-correlation functions $\left\langle n_{\uparrow}\left(\mathbf{r}_{0}\right) n_{\downarrow}(\mathbf{r})\right\rangle$, with $\mathbf{r}_{0}$ denoted by a (yellow) marker. Phase plot (b4) is derived from reduced wave function $\psi_{c, \downarrow}(\mathbf{r})$, which is computed by fixing seven of the eight particles at their most probable locations, and their corresponding radii are indicated by (yellow) markers. The closed dashed (blue) contour is a guide to the eye, which allows us to count the number of phase slips.

observe that with increasing interaction strengths, the minima of the entanglement pseudoenergies $\xi_{i}$ shifts to larger $J_{z}^{A} / N$ values. To illustrate the internal structure and the correlations between the up-spin and down-spin components of these $\mathcal{P} \mathcal{T}$ eigenstates, we show the pair-correlation functions in Figs. 9(c4) and 9(d4).

With our understanding of the OES plots in Figs. 9, we may now explain various features observed in the EE plots that help us understand the correlation properties of the ground states in the mean-field-like and strongly correlated regimes. As discussed with reference to Figs. 4(b) and 5(b), we observe qualitatively similar features in the $N=8$ particle case as well. The presence of distinctly different slopes in Figs. 7(b) and 8 (b) suggests the presence of distinct correlation properties in different ground states within various phases. Within each phase, EE increases monotonously with increasing $g$ due to the presence of increased correlations in the ground state. For example, to illustrate this feature within the $\mathcal{P} \mathcal{T}(\mathrm{SC})$ phase, we may compare the OES plots in Figs. 9(c3) and 9(d3) and observe an increased homogeneity in the Fock states. As a side note, we observe a small region of $\mathcal{P}$-symmetric states before the transition to the strongly correlated regime. These states do not possess distinct topological or correlation properties. Without loss of generality, we assert that these ground states merely occupy a crossover region prior to the transition to the strongly correlated regime.

In summary, we emphasize that the ground states in the weakly interacting regime illustrated in the top two rows of Fig. 9 are mean-field-like states. Their density distributions, pair-correlation functions, and reduced wave functions may be readily related to the results from mean-field theory computations discussed in our earlier paper [30]. Within the ED scheme, we even reproduce the reversal of phase symmetry between $\mathcal{P}$ and $\mathcal{P} \mathcal{T}$ eigenstates that is observed with an increasing value of $g$, but with a fixed value of $g_{\uparrow \downarrow} / g$ in our earlier mean-field study [30]. Such a correspondence between 
ED results and mean-field theory results is anticipated only when the ground state is predominantly occupied by one single-particle eigenstate (and/or its time-reversal partner), as revealed in Figs. 9(a2) and 9(b2). As illustrated in the bottom two rows of Fig. 9, the presence of a large degeneracy in entanglement pseudoenergies and the distribution of particles across many single-particle eigenstates are clear manifestations of the strongly correlated nature of the ground states. Furthermore, we observe from Figs. 9 that the transition from a mean-fieldlike regime to a strongly correlated regime is attained with only small variations in the magnitudes of interparticle interaction strengths. We emphasize here that the pivotal reason behind this feature is the presence of a nearly flat single-particle energy spectrum at large SO-coupling strengths.

\section{CONCLUSIONS}

We systematically study an interacting few-body system of two-component Bose gases with 2D isotropic Rashba SO-coupling in a 2D isotropic harmonic trap. We show that the model Hamiltonian is gauge equivalent to particles subject to a $\mathcal{T}$-symmetry preserving pure non-Abelian vector potential, whose magnitude proportionally determines the strength of Rashba SO coupling. It is experimentally feasible to devise a scheme in which tunable parameters, such as laser fields, can be used to control the magnitude of a non-Abelian vector potential, and hence simulate large SO-coupling strengths. In this limit of large SO-coupling strengths, we show that the single-particle energy spectrum is nearly flat. In the recent past, several research groups have made proposals to engineer quantum systems in which interactions would play a dominant role and the ground states would, in turn, be strongly correlated. For example, recent proposals suggest schemes that would engineer nearly flat Chern bands to study strongly correlated fractional quantum Hall states in the lattice limit [32]. Though we study few-body Bose gases in traps, we emphasize that the intention with which we have identified the existence of nearly flat energy spectra at large SO-coupling strengths is not too dissimilar from the aforementioned line of thought.

In our model system with nearly flat energy spectra, we observe that the presence of interparticle interactions allows for the emergence of ground states with distinct topological, symmetry, and correlation properties. We solve the interacting Hamiltonian in different particle-number scenarios and analyze the ground-state properties with the help of energy spectrum, the single-particle density matrix, pair-correlation functions, reduced wave functions, and entanglement measures. At small particle numbers, we show the phase diagram in Figs. 4 and 5, with ground states being eigenstates of either the $\mathcal{P}$ or $\mathcal{P} \mathcal{T}$ operator. In Fig. 6, we illustrate the ground-state properties at representative interaction strengths in various phases. We further assert that the bosons condense to an array of topological $\mathcal{P}$ eigenstates with $n+1 / 2$ quantum angular momentum vortex configuration, with $n=0,1,2,3$. At large particle numbers, we illustrate the phase diagram in Figs. 7 and 8. We observe the presence of two distinct regimes: (a) at weak-interaction strengths (mean-field-like regime), we obtain ground states with topological and symmetry properties that are also obtained via mean-field theory computations (We justify this correspondence and illustrate the ground-state properties in detail in Fig. 9.); and (b) at intermediate- to strong-interaction strengths (strongly correlated regime), we report the emergence of strongly correlated ground states. The properties illustrated in Fig. 9 demonstrate the correlated nature of the ground states.

In recent years, there has been tremendous experimental progress in realizing few-particle systems in a trap setting [33] or in an optical lattice potential [34]. The significance of these experiments to various ED studies cannot be overemphasized. Such seminal experiments have the potential to transform studies of few-particle systems from simple model systems to novel stand-alone systems with intrinsic value $[35,36]$.

It is interesting to inquire whether the strongly correlated ground states that emerge in the nearly flat energy spectra would eventually allow for the manifestation of bosonic analogues of topological insulators predicted to occur in traditional condensed-matter systems. We emphasize that in our system of trapped bosons, quantum statistics makes it impossible to fill up the lowest generalized Landau level. This results in the absence of "sharp boundaries," which, in turn, obviates the occurrence of states with topological order. However, this fundamental roadblock may be circumvented when we consider a system of SO-coupled bosons or fermions in specially engineered optical lattices [37].

Note added. Recently, Tigran A. Sedrakyan and co-workers reported the possible emergence of an interesting composite fermion ground state in a spinor Bose gas with the isotropic Rashba spin-orbit coupling in two dimensions [38].

\section{ACKNOWLEDGMENTS}

B.R. thanks L. O. Baksmaty, C. Zhang, H. Lu, and L. Dong for useful discussions. B.R. and H.P. acknowledge support by the NSF (Grant No. PHY-1205973), the Welch Foundation (Grant No. C-1669), and the DARPA OLE program. H.H. was supported by the ARC Discovery Project No. DP0984522.
[1] I. Bloch, J. Dalibard, and W. Zwerger, Rev. Mod. Phys. 80, 885 (2008); M. Inguscio, W. Ketterle, and C. Salomon, Ultra-cold Fermi Gases (IOS, Amsterdam, 2008); M. Lewenstein et al., Adv. Phys. 56, 243 (2007).

[2] R. Onofrio, C. Raman, J. M. Vogels, J. R. Abo-Shaeer, A. P. Chikkatur, and W. Ketterle, Phys. Rev. Lett. 85, 2228 (2000); R. Desbuquois et al., Nature Phys. 8, 645 (2012); M. W. Zwierlein et al., Nature (London) 435, 1047 (2005).

[3] S. E. Pollack, D. Dries, and R. G. Hulet,
Science 326, 5960 (2009); J. R. Williams, E. L. Hazlett, J. H. Huckans, R. W. Stites, Y. Zhang, and K. M. OHara, Phys. Rev. Lett. 103, 130404 (2009).

[4] M. Greiner et al., Nature (London) 415, 39 (2002); U. Schneider et al., Science 322, 1520 (2008); R. Jördens et al., Nature (London) 455, 204 (2008).

[5] P. M. Duarte, R. A. Hart, J. M. Hitchcock, T. A. Corcovilos, T. L. Yang, A. Reed, and R. G. Hulet, Phys. Rev. A 84, 061406(R) (2011). 
[6] J. Struck et al., Science 333, 996 (2011).

[7] Y.-J. Lin et al., Nature (London) 462, 628 (2009); Nature Phys. 7, 531 (2011).

[8] J. Dalibard et al., Rev. Mod. Phys. 83, 1523 (2011).

[9] Y.-J. Lin et al., Nature (London) 471, 83 (2011).

[10] P. Wang, Z. Q. Yu, Z. Fu, J. Miao, L. Huang, S. Chai, H. Zhai, and J. Zhang, Phys. Rev. Lett. 109, 095301 (2012); L. W. Cheuk, A. T. Sommer, Z. Hadzibabic, T. Yefsah, W. S. Bakr, and M. W. Zwierlein, ibid. 109, 095302 (2012).

[11] C. L. Kane and E. J. Mele, Phys. Rev. Lett. 95, 226801 (2005); B. A. Bernevig and S.-C. Zhang, ibid. 96, 106802 (2006).

[12] M. Burrello and A. Trombettoni, Phys. Rev. A 84, 043625 (2011); B. Juliá-Díaz et al., New J. Phys. 14, 055003 (2012).

[13] D. S. Petrov, M. Holzmann, and G. V. Shlyapnikov, Phys. Rev. Lett. 84, 2551 (2000).

[14] T. Yefsah, R. Desbuquois, L. Chomaz, K. J. Gunter, and J. Dalibard, Phys. Rev. Lett. 107, 130401 (2011).

[15] M. Burrello and A. Trombettoni, Phys. Rev. Lett. 105, 125304 (2010).

[16] Y. Li, X. Zhou, and C. Wu, Phys. Rev. B 85, 125122 (2012).

[17] Z. F. Xu and L. You, Phys. Rev. A 85, 043605 (2012).

[18] B. Ramachandhran, B. Opanchuk, X. J. Liu, H. Pu, P. D. Drummond, and H. Hu, Phys. Rev. A 85, 023606 (2012).

[19] J. M. Zhang and R. X. Dong, Eur. J. Phys. 31, 591 (2010). Techniques illustrated in this paper were particularly useful during implementation.

[20] L. O. Baksmaty, C. Yannouleas, and U. Landman, Phys. Rev. A 75, 023620 (2007); B. Juliá-Díaz, D. Dagnino, K. J. Gunter, T. Grass, N. Barberan, M. Lewenstein, and J. Dalibard, ibid. 84, 053605 (2011).

[21] N. Barberán, M. Lewenstein, K. Osterloh, and D. Dagnino, Phys. Rev. A 73, 063623 (2006).

[22] H. Saarikoski et al., Europhys. Lett. 91, 30006 (2010).

[23] Y. Shi, Phys. Rev. A 67, 024301 (2003); J. Phys. A 37, 6807 (2004).
[24] A. Sterdyniak, B. A. Bernevig, N. Regnault, and F. D. M. Haldane, New J. Phys. 13, 105001 (2011).

[25] H. Li and F. D. M. Haldane, Phys. Rev. Lett. 101, 010504 (2008).

[26] O. S. Zozulya, M. Haque, and N. Regnault, Phys. Rev. B 79, 045409 (2009).

[27] C. Wu, I. Mondragon-Shem, and X.-F. Zhou, Chin. Phys. Lett. 28, 097102 (2011).

[28] M. M. Salomaa and G. E. Volovik, Phys. Rev. Lett. 55, 1184 (1985).

[29] C. Wang, C. Gao, C.-M. Jian, and H. Zhai, Phys. Rev. Lett. 105, 160403 (2010).

[30] H. Hu, B. Ramachandhran, H. Pu, and X.-J. Liu, Phys. Rev. Lett. 108, 010402 (2012).

[31] In Fig. 5(b), the $\mathcal{P} 2 \rightarrow \mathcal{P} 3$ transition neither exhibits a change in slope nor a noticeable drop in EE value. From an analysis of the OES plots across this transition (not shown), we observe that the maximally contributing entanglement pseudoenergies $\xi_{i}$ at $J_{z}^{A}=1.5(\mathcal{P} 2)$ and $J_{z}^{A}=2.5(\mathcal{P} 3)$ are nearly degenerate, and hence we observe this anomaly. In a broader sense, we conclude that the ground states with $\mathcal{P} 2$ symmetry in Fig. 5(b) may merely occupy a small crossover region between the $\mathcal{P} \mathcal{T}$ and $\mathcal{P} 3$ phases.

[32] R. Roy and S. L. Sondhi, Physics 4, 46 (2011); T. Neupert, L. Santos, C. Chamon, and C. Mudry, Phys. Rev. Lett. 106, 236804 (2011); E. Tang, J.-W. Mei, and X.-G. Wen, ibid. 106, 236802 (2011); K. Sun, Z. Gu, H. Katsura, and S. DasSarma, ibid. 106, 236803 (2011).

[33] F. Serwane et al., Science 332, 336 (2011).

[34] N. Gemelke, E. Sarajlic, and S. Chu, arXiv:1007.2677.

[35] H. Saarikoski, S. M. Reimann, A. Harju, and M. Manninen, Rev. Mod. Phys. 82, 2785 (2010).

[36] Nigel R. Cooper and Jean Dalibard, arXiv:1212.3552.

[37] T. D. Stanescu, V. Galitski, J. Y. Vaishnav, C. W. Clark, and S. DasSarma, Phys. Rev. A 79, 053639 (2009). In this reference, the authors propose to realize topological phases emerging from a single-particle Hamiltonian in optical lattices.

[38] Tigran A. Sedrakyan, Alex Kamenev, and Leonid I. Glazman, Phys. Rev. A 86, 063639 (2012). 